\title{
A AFECÇÃO VESTIBULAR INFANTIL: ESTUDO DA ORIENTAÇÃO ESPACIAL
}

\section{Vestibular disorders in childhood: study of spatial disorders}

\author{
Elaine Shizue Novalo ${ }^{(1)}$, Maria Valéria Schmidt Goffi-Gomez ${ }^{(2)}$, \\ Ítalo Roberto Torres de Medeiros ${ }^{(3)}$, Maria Elisabete Bovino Pedalini ${ }^{(4)}$, \\ Rosa Maria Rodrigues dos Santos ${ }^{(5)}$
}

\section{RESUMO}

Objetivo: verificar como é a percepção do espaço na criança com vertigem periférica. Métodos: estudo prospectivo de 18 crianças, com faixa etária de três a 15 anos, sob acompanhamento no Ambulatório de Otorrinolaringologia / Hospital das Clínicas da Faculdade de Medicina da Universidade de São Paulo. A percepção espacial foi avaliada por meio de atividade livre (desenho livre) e dirigida (blocos lógicos e teste de Frostig). Das 18 crianças avaliadas, nove constituíram o grupo estudo (diagnóstico de vestibulopatia e queixa de tontura) e nove constituíram o grupo controle (sem história de vestibulopatia e sem queixa de tontura). Foram excluídas crianças que apresentassem comprometimento de Sistema Nervoso Central que interferisse na interpretação da avaliação. Os resultados obtidos foram comparados entre o grupo estudo e controle qualitativamente. Resultados: em relação ao desenho livre, $77,78 \%$ das crianças do grupo controle e $55,55 \%$ do grupo estudo utilizaram o papel inteiro. Quanto à proporção dos objetos, $100 \%$ das crianças do grupo controle e apenas $48,86 \%$ das crianças do grupo estudo desenharam com proporção adequada. Nos blocos lógicos, houve maior dificuldade do grupo estudo. No teste de Frostig, foi observada diferença significativa do ponto de vista clínico entre os grupos quanto ao Quociente Perceptual. Conclusão: as crianças com vestibulopatia, ao desenhar, aproveitaram o papel de forma menos homogênea, com maior dificuldade para desenhar figuras humanas e proporção inadequada entre os objetos. Desta forma, é importante que seja realizado o diagnóstico de vestibulopatia precocemente, para que o tratamento/reabilitação seja iniciado e sintomas, como a desorientação espacial, não influenciem na aprendizagem da criança.

DESCRITORES: Tontura; Vertigem; Criança; Percepção Espacial

(1) Fonoaudióloga; Colaboradora do Setor de Reabilitação Vestibular do Hospital das Clínicas da Faculdade de Medicina da Universidade de São Paulo; Especialização em Audiologia pela Irmandade da Santa Casa de Misericórdia de São Paulo.

(2) Fonoaudióloga; Responsável pelo Setor de Fonoaudiologia do Grupo de Implante Coclear do Hospital das Clínicas da Faculdade de Medicina da Universidade de São Paulo; Doutora em Distúrbios da Comunicação Humana pela Universidade Federal de São Paulo.

(3) Médico Otorrinolaringologista; Responsável pelo Setor de Vertigem na Infância do Hospital das Clínicas da Faculdade de Medicina da Universidade de São Paulo; Doutor em Medicina pela Disciplina de Otorrinolaringologia da Faculdade de Medicina da Universidade de São Paulo.

(4) Fonoaudióloga; Responsável pelo Setor de Reabilitação Vestibular do Hospital das Clínicas da Faculdade de Medicina da Universidade de São Paulo; Doutora em Ciências Médicas pela Fisiopatologia Experimental da Faculdade de Medicina da Universidade de São Paulo.

(5) Psicóloga; Responsável pelo Setor de Psicologia do Ambulatório de Otorrinolaringologia do Hospital das Clínicas da Faculdade de Medicina da Universidade de São Paulo.

\section{INTRODUÇÃO}

O equilíbrio corporal pode ser definido como a capacidade do ser humano em manter-se ereto e executar movimentos do corpo sem oscilações ou quedas. Esse complexo fenômeno sensório-motor é gerado pela integração de informações de três sistemas sensoriais (sistema visual, somatossensorial e vestibular) no sistema nervoso central. Alterações na manutenção do equilíbrio corporal podem ocasionar em sintomas como vertigem, tontura e desequilíbrio ${ }^{1-8}$.

A vestibulopatia na infância é de difícil diagnóstico, tanto pela subjetividade dos sintomas e sinais, quanto pela dificuldade da criança em descrever e relatar seus sintomas sendo, por isso, registrada em menor quantidade ${ }^{5,9-21}$.

Os sintomas provocados pelos distúrbios vestibulares dificultam as relações espaciais e o ade- 
quado contato com o meio ambiente, comprometendo a aprendizagem da criança e sua habilidade de comunicação. Além do retardo na aquisição da linguagem (maior facilidade de compreensão do que de expressão), as crianças com vestibulopatias periféricas podem apresentar retardo motor, atraso para ficar na posição ereta e andar 22-32.

Em crianças de idade escolar, esses sintomas podem influenciar algumas fases do desenvolvimento infantil, gerando alterações de postura corporal, equilíbrio físico e coordenação motora, que são fundamentais na aquisição do aprendizado da linguagem oral e escrita ${ }^{33}$.

Essas dificuldades de aprendizagem em crianças com distúrbios vestibulares podem ser explicadas pela inabilidade para realizar movimentos coordenados e a concepção imprecisa de sua própria posição espacial, já que o sistema postural atua sobre os mecanismos de aprendizagem por meio de integrações sensoriais provenientes fundamentalmente de aferências proprioceptivas, vestibulares e visuais. O sistema vestibular permite, direta ou indiretamente, a percepção da noção espacial e temporal nas sucessivas etapas do desenvolvimento ${ }^{33-36}$.

Além disso, os distúrbios vestibulares na infância estão associados ao desenvolvimento de reações emocionais e alterações comportamentais que podem prejudicar a inserção da criança em seu meio social ${ }^{37}$.

Considerando que a vestibulopatia infantil pode ser seguida dos mais diversos sintomas e que, estes sintomas podem interferir no desenvolvimento normal da criança, pode ser de grande importância observar como a criança portadora de vestibulopatia possui a percepção do espaço ${ }^{38}$.

Sendo assim, este estudo teve como finalidade verificar como é a percepção do espaço na criança com vertigem periférica.

\section{MÉTODOS}

Foi realizado um estudo prospectivo de 18 crianças, com faixa etária entre três e 15 anos de idade, sendo 11 do sexo feminino e sete do sexo masculino. Todas as crianças foram avaliadas, durante os anos de 2004 e 2005, no Setor de Fonoaudiologia da Divisão de Clínica Otorrinolaringológica do Hospital das Clínicas da Faculdade de Medicina da Universidade de São Paulo. O protocolo de pesquisa foi apresentado aos responsáveis pelas crianças que assinaram o termo de consentimento.

Os pacientes foram divididos em dois grupos pareados pela idade: grupo estudo e controle. O grupo estudo foi constituído por nove crianças com afecções vestibulares periféricas e queixa de ton- tura, encaminhadas pelo Setor de Otoneurologia Infantil; e o grupo controle, por nove crianças sem histórico de afecções vestibulares e queixa de tontura, voluntárias convidadas do Setor de Motricidade Oral.

Foram excluídas crianças com histórico ou sintomas de comprometimento no sistema nervoso central que alterassem o desempenho ou a interpretação da avaliação.

Primeiramente, foi aplicado um questionário (Figura 1) nos responsáveis, para verificar o desenvolvimento neuro-psico-motor, de linguagem e escolar, além de sintomas e atividades lúdicas da preferência da criança. A avaliação da orientação espacial propriamente dita ocorreu em três partes:

\section{1a Parte}

- Atividade livre: Desenho sem tema definido

Foi entregue à criança uma folha de papel sulfite, em branco, do tipo A4 e lápis de colorir, em seguida, solicitou-se a ela que realizasse um desenho de sua preferência.

\section{Parte}

- Atividade dirigida: Avaliação por meio de blocos lógicos

Foram utilizados 24 blocos lógicos com formas geométricas de círculo e quadrado, que se distinguiam pelos aspectos de espessura, tamanho e cor (Figura 2 - adaptado de De Renzi e Vignolo, 1962) ${ }^{39}$. Durante as tarefas, era solicitada a criança que localizasse as formas geométricas de acordo com o aspecto analisado.

\section{3ㅁ Parte}

- Atividade dirigida: Teste de Frostig

Utilização do Teste de Frostig para verificar habilidades como coordenação motora-visual, discriminação de figura-fundo, constância de forma, posição espacial e relações espaciais ${ }^{26}$.

A análise dos dados considerou as variáveis a seguir:

\section{Questionário:}

- Desenvolvimento neuro-psico-motor:

- Idade (em meses) para sentar, engatinhar, andar

- Desenvolvimento de linguagem oral:

- Idade (em meses) para o aparecimento das primeiras palavras

- Comportamento da criança:

- Tranqüila X Agitada

- Sintomas e características relacionadas à síndrome vestibular:

- Descrição dos sinais e sintomas da criança 


\section{AFECÇÃO VESTIBULAR INFANTIL: ESTUDO DA ORIENTAÇÃO ESPACIAL QUESTIONÁRIO}

Nome: data:

Idade: $R G(H C)$

Nome da mãe: telefone:

Desenvolvimento motor

Idade em que sentou:

Idade em que engatinhou:

Idade em que andou:

Desenvolvimento de linguagem

Idade em que compôs as primeiras palavras:

Comportamento

Calma ( ) Agitada ( )

Medo de escuro:

Urina enquanto dorme:

Sinais e sintomas

Dor de barriga ( ) Cefaléia ( )

Náuseas/vômitos ( ) Diarréia ( )

Cinetose ( ) Quedas freqüentes ( )

Outros:

Sensação subjetiva de acuidade auditiva

Adequada ( ) Inadequada ( )

Atividades físicas / Lúdicas

Anda de bicicleta? Sim ( ) Não ( )

Brincadeira preferida:

Escolaridade

Série que cursa atualmente:

Desempenho escolar

Repetição de ano letivo

Maiores informações:

Figura 1 - Questionário 


\section{AFECÇÃO VESTIBULAR INFANTIL: ESTUDO DA ORIENTAÇÃO ESPACIAL BLOCOS LÓGICOS}

Nome: data:

Idade: $R G(H C)$ :

Nome da mãe: telefone:

$11^{\mathrm{a}}$ parte (preparação)

6 blocos: círculos e quadrados grandes (amarelo, azul e vermelho)

\begin{tabular}{|l|||l||l|}
\hline 1. & Pegue o círculo amarelo \\
\hline 2. & Pegue o quadrado azul & \\
\hline 3. & Pegue o círculo vermelho & \\
\hline
\end{tabular}

9 blocos: círculos grandes, pequenos e quadrados grandes (amarelo, azul e vermelho)

\begin{tabular}{||l||l|l|}
\hline 4. $\quad$ Pegue o círculo amarelo pequeno \\
\hline 5. & Pegue o quadrado azul grande \\
\hline 6. Pegue o círculo vermelho grande \\
\hline
\end{tabular}

\section{$\underline{2^{\mathrm{a}} \text { parte (teste de tamanho) }}$}

12 blocos: círculos e quadrados, grandes e pequenos (amarelo, azul e vermelho)

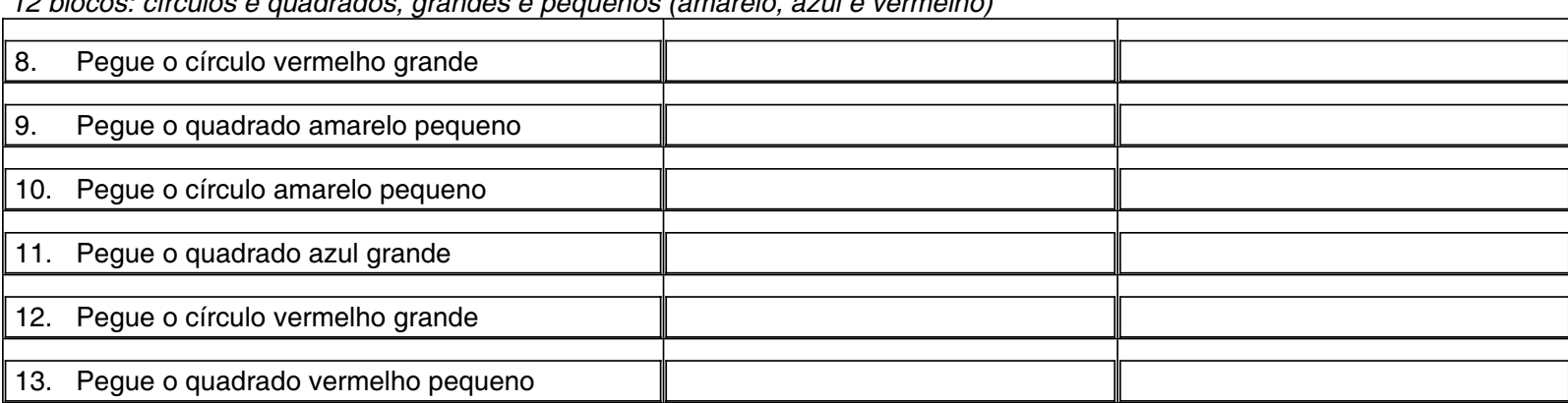

\section{$\underline{3^{\mathrm{a}} \text { parte (teste de espessura) }}$}

12 blocos: círculos e quadrados, finos e grossos (amarelo, azul e vermelho)

\begin{tabular}{|l||||||||}
\hline 14. Pegue o círculo azul fino \\
\hline 15. Pegue o círculo amarelo grosso \\
\hline 16. Pegue o quadrado azul fino \\
\hline 17. Pegue o círculo vermelho fino \\
\hline \hline 18. Pegue o quadrado amarelo grosso
\end{tabular}

\section{Figura 2 - Avaliação por meio de blocos lógicos}


$\underline{4^{\mathrm{a}} \text { parte (teste de espessura e tamanho) }}$

24 blocos: círculos e quadrados, grandes e pequenos, finos e grossos (amarelo, azul e vermelho)

\begin{tabular}{|l|||l|}
\hline 20. Pegue o círculo azul grosso grande & \\
\hline 21. Pegue o círculo amarelo fino pequeno \\
\hline 22. Pegue o quadrado azul grosso pequeno \\
\hline 23. Pegue o círculo vermelho fino grande & \\
\hline 24. Pegue o quadrado amarelo grosso pequeno & \\
\hline 25. Pegue quadrado vermelho grosso grande & \\
\hline 26. Pegue o círculo azul fino pequeno \\
\hline 2 27. Pegue o quadrado azul grosso grande \\
\hline
\end{tabular}

\section{$5^{\mathrm{a}}$ parte (teste de espessura e tamanho)}

12 blocos: círculos grandes e pequenos, finos e grossos (amarelo, azul e vermelho)

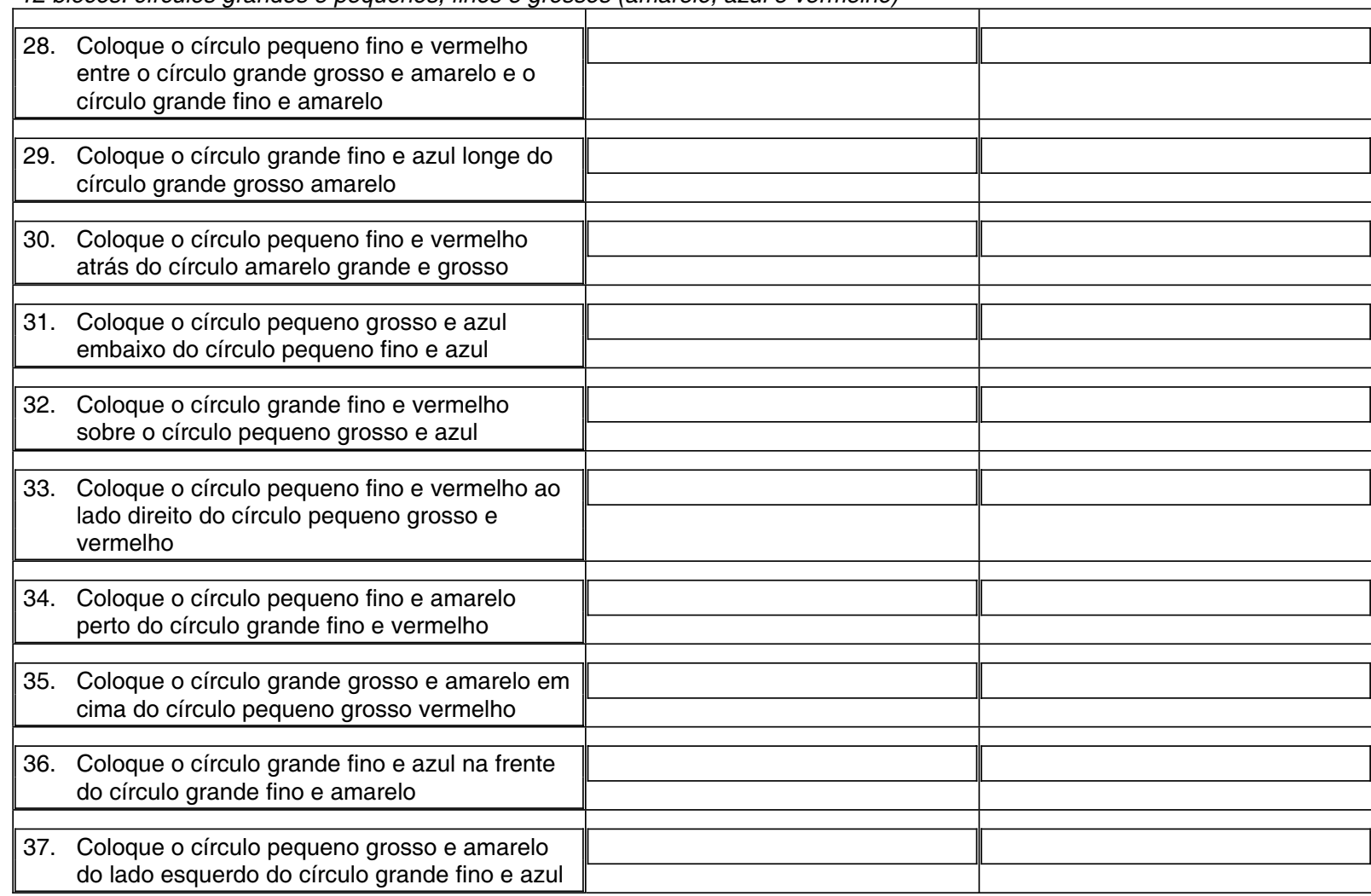

\section{Figura 2 (cont.) - Avaliação por meio de blocos lógicos}

- Sensação subjetiva de acuidade auditiva:

- Adequada X Inadequada

- Comportamento escolar:

- Satisfatório (Sem dificuldades) X Insatisfatório

(Presença de dificuldades)

- Atividade lúdica de preferência:

- Enquadrar a atividade lúdica de preferência da criança em atividades que exigem movimentação corporal ou atividades lúdicas que não exigem movimentação corporal.
Atividade livre:

- Posição do papel utilizada:

Horizontal ou Vertical;

- Aproveitamento do papel:

Espaço do papel em que houve maior predominância do desenho - centro, inteiro, quadrante, bordas;

- Figura humana:

Nos casos em que a criança optou por realizar o desenho de figura humana, analisou-se a propor- 
ção e a presença das partes que constituem o corpo humano. Foi considerada como adequada, a figura humana que possuía proporção e presença das partes constituintes do corpo humano como, por exemplo, membros inferiores, superiores, tronco e cabeça; inadequada, a figura humana sem proporção entre as partes do corpo ou que faltava algum membro do corpo humano;

- Proporção dos objetos no desenho:

Foi analisada a proporção entre os objetos contidos no desenho da criança, ou seja, verificou-se o nível de distância e tamanho entre todos eles, considerando como adequado, o desenho que possuía tamanho e distância semelhante à realidade $\mathrm{e}$, inadequado, aquele em que os objetos possuíam tamanho e distância destoantes da realidade, consideradas as idades das crianças.

\section{Blocos lógicos:}

- Preparação (ordens simples com a finalidade de familiarizar a criança com o teste);

- Percepção de tamanho;

- Percepção de espessura;

- Percepção de tamanho e espessura;

- Percepção de tamanho, espessura e posição espacial.

Para análise cautelosa e pormenorizada dos dados, nos casos em que a criança errou algum item durante a avaliação, a tarefa analisada (tamanho; espessura; tamanho e espessura; tamanho, espessura e localização espacial) foi repetida mais uma vez.
Teste de Frostig:

- Coordenação motora e visual;

- Discriminação de figura-fundo;

- Constância de forma;

- Posição espacial;

- Relações espaciais.

Este trabalho foi previamente apresentado e aceito pela Comissão de Ética para análise de projetos de pesquisa (CAPPesq) do Hospital das Clínicas da Faculdade de Medicina da Universidade de São Paulo sob protocolo número 187/98.

Para análise objetiva entre os grupos, foi avaliado o Raw Score de cada teste (pontuação obtida em cada um dos itens analisados) e o Quociente Perceptual - PQ (somatória de toda a pontuação obtida nos itens analisados correlacionados com a idade da criança), proposto por Quirós (1973) ${ }^{17}$.

Os resultados obtidos em todas as etapas da avaliação dos pacientes foram comparados entre o grupo estudo e controle qualitativamente.

\section{RESULTADOS}

A distribuição da amostra entre os dois grupos mostrou ser similar no que se refere à idade (Tabela 1), embora o sexo feminino tenha sido predominante no grupo estudo (Tabela 2).

Observando a Tabela 3, pode-se verificar que não houve diferenças significativas do ponto de vista clínico, quanto ao desenvolvimento motor das crianças do grupo estudo com as do grupo controle.

Tabela 1 - Faixa etária das crianças avaliadas em ambos os grupos estudados

\begin{tabular}{cccc}
\hline Idade & $\begin{array}{c}\text { Grupo Estudo } \\
\mathrm{n}(\%)\end{array}$ & $\begin{array}{c}\text { Grupo Controle } \\
\mathrm{n}(\%)\end{array}$ & $\begin{array}{c}\text { Total } \\
\mathrm{n}(\%)\end{array}$ \\
\hline $3 \mid-5$ & $1(5,55)$ & $1(5,55)$ & $2(11,1)$ \\
$5 \mid-7$ & $1(5,55)$ & $1(5,55)$ & $2(11,1)$ \\
$7 \mid-9$ & $0(0)$ & $0(0)$ & $0(0)$ \\
$9 \mid-11$ & $3(16,67)$ & $3(16,67)$ & $6(33,34)$ \\
$11 \mid-13$ & $3(16,67)$ & $2(11,11)$ & $5(27,78)$ \\
$13 \mid-$ & $1(5,55)$ & $2(11,11)$ & $3(16,66)$ \\
Total & $9(50)$ & $9(50)$ & $18(100)$ \\
\hline
\end{tabular}

Tabela 2 - Distribuição das crianças em relação ao sexo em ambos os grupos estudados

\begin{tabular}{cccc}
\hline Sexo & $\begin{array}{c}\text { Grupo Estudo } \\
\mathrm{n}(\%)\end{array}$ & $\begin{array}{c}\text { Grupo Controle } \\
\mathrm{n}(\%)\end{array}$ & $\begin{array}{c}\text { Total } \\
\mathrm{n}(\%)\end{array}$ \\
\hline Masculino & $2(11,11)$ & $5(77,78)$ & $7(38,88)$ \\
Feminino & $7(38,88)$ & $4(22,22)$ & $11(61,12)$ \\
\hline Total & $9(50)$ & $9(50)$ & $18(100)$ \\
\hline
\end{tabular}


Tabela 3 - Distribuição das crianças quanto ao desenvolvimento motor (em meses) em ambos os grupos estudados

\begin{tabular}{lcccccc}
\hline \multirow{2}{*}{ Sujeito } & \multicolumn{3}{c}{ Grupo Estudo } & \multicolumn{3}{c}{ Grupo Controle } \\
\cline { 2 - 7 } & Sentar & Engatinhar & Andar & Sentar & Engatinhar & Andar \\
\hline 1 & 06 & $-^{*}$ & 13 & $-{ }^{*}$ & $-{ }^{*}$ & 18 \\
2 & 09 & $-{ }^{*}$ & 14 & 12 & 15 & 18 \\
3 & 12 & 24 & 36 & 07 & 07 & 18 \\
4 & 05 & 08 & 11 & 06 & 07 & 12 \\
5 & 05 & 06 & 12 & 05 & 09 & 14 \\
6 & 11 & 10 & 14 & 07 & 05 & 11 \\
7 & 08 & 06 & 18 & 08 & $-*$ & 30 \\
8 & 06 & 07 & 12 & 12 & 24 & 15 \\
9 & 10 & 10 & 16 & 12 & 11 & 16,37 \\
\hline Média & 08 & 10,14 & 16,22 & 8,62 & 11,14 & 14 \\
\hline
\end{tabular}

Tabela 4 - Distribuição das crianças quanto à aquisição de linguagem oral (em meses)

\begin{tabular}{ccc}
\hline & Grupo Estudo & Grupo Controle \\
\hline 1 & 18 & ${ }^{*}$ \\
2 & 14 & 24 \\
3 & 36 & 08 \\
4 & 12 & 16 \\
5 & 09 & 12 \\
6 & 24 & 18 \\
7 & 24 & 10 \\
8 & 12 & 36 \\
9 & 13 & 15 \\
\hline Média & 18 & 17,37 \\
\hline
\end{tabular}

Um fato que dificultou a análise deste item foi que muitos pais, ou responsáveis, não conseguiram referir a idade em que a criança iniciou as atividades motoras analisadas.

Também não se observam diferenças significativas do ponto de vista clínico, quanto ao desenvolvimento de linguagem dessas crianças (Tabela 4).

Em relação ao acompanhamento escolar (Tabela 5), duas crianças do grupo estudo foram destacadas pelos pais quanto às dificuldades de acompanhamento escolar, porém nenhuma delas apresentou repetição de ano letivo.

Quanto às atividades lúdicas de preferência (Tabela 5), observou-se que três crianças do grupo estudo preferem atividades de movimento (como jogar bola, brincar de roda, correr, andar de bicicleta, praticar esportes, entre outras) e seis preferem atividades sem movimento (como assistir televisão, brincar de boneca, videogame, leitura de livros, entre outros). Em relação ao grupo controle, quatro crianças relataram que preferem atividades de movimento e cinco preferem atividades sem movimento. Nos dois grupos verificou-se que as atividades sem movimento foram preferidas pelas crianças, além disso, observou-se que em ambos os grupos, algumas crianças consideraram a leitura como atividade preferida.

No que diz respeito à sensação subjetiva de acuidade auditiva (Tabela 5), apenas uma criança do grupo controle e do grupo estudo foi apontada pelos pais com presença de queixa de hipoacusia.

Analisando a Figura 3, pode-se verificar que não houve diferença quanto à posição do papel utilizada durante o desenho, sendo que tanto no grupo estudo quanto no grupo controle, sete crianças $(77,78 \%)$ utilizaram o papel na posição horizontal para desenhar e duas crianças $(22,22 \%)$ utilizaram o papel na posição vertical.

Em relação ao aproveitamento do papel como mostra a Figura 4, no grupo estudo, uma criança $(11,11 \%)$ realizou o desenho na parte central da folha de papel sulfite, cinco crianças $(55,55 \%)$ utilizaram o papel inteiro para desenhar, duas crianças $(22,22 \%)$ utilizaram apenas quadrantes do papel e uma criança $(11,11 \%)$ utilizou as bordas para realizar o desenho. Já no grupo controle, pode-se verificar que sete crianças $(77,78 \%)$ utilizaram o papel inteiro para realizar o desenho sendo que, apenas duas crianças $(22,22 \%)$ utilizaram quadrantes para realizar seus desenhos.

No que diz respeito ao desenho da figura humana, no grupo estudo apenas uma criança realizou desenho de figura humana e este foi considerado alterado; já no grupo controle, sete crianças $(100 \%)$ realizaram desenho de figura humana de forma adequada. 
Tabela 5 - Perfil das crianças estudadas de acordo com o questionário aplicado

\begin{tabular}{cccc}
\hline & $\begin{array}{c}\text { Grupo Estudo } \\
\mathbf{n}(\%)\end{array}$ & $\begin{array}{c}\text { Grupo Controle } \\
\mathbf{n}(\%)\end{array}$ & $\begin{array}{c}\text { Total } \\
\mathbf{n}(\%)\end{array}$ \\
\hline $\begin{array}{c}\text { Desenvolvimento Escolar } \\
\text { Satisfatório }\end{array}$ & $7(88,89)$ & $8(94,45)$ & $15(83,34)$ \\
Insatisfatório & $2(11,11)$ & $0(0)$ & $2(11,11)$ \\
Repetição & $0(0)$ & $1(5,55)$ & $1(5,55)$ \\
\hline Total & $9(50)$ & $9(50)$ & $18(100)$ \\
\hline Atividade Lúdica & & & $7(38,89)$ \\
Atividades com Movimento * & $3(16,66)$ & $4(22,23)$ & $11(61,11)$ \\
Atividades sem Movimento * & $6(33,34)$ & $5(27,77)$ & $18(100)$ \\
\hline Total & $9(50)$ & $9(50)$ & \\
\hline $\begin{array}{c}\text { Sensação Subjetiva de } \\
\text { Acuidade Auditiva }\end{array}$ & & & $16(88,88)$ \\
Adequada & $8(44,44)$ & $8(44,44)$ & $2(11,12)$ \\
Inadequada & $1(5,56)$ & $1(5,56)$ & $18(100)$ \\
\hline Total & $9(50)$ & $9(50)$ &
\end{tabular}

* Vide definição presente em Método

Posição do Papel

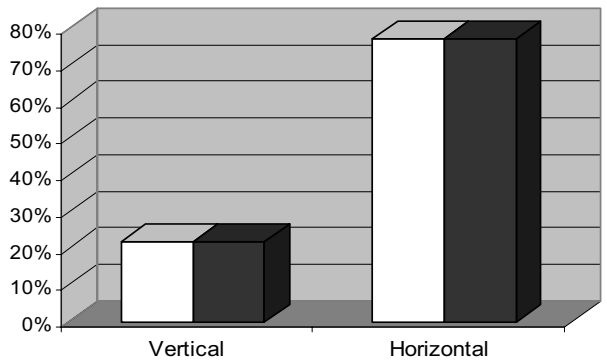

Figura 3 - Distribuição das crianças quanto à posição do papel

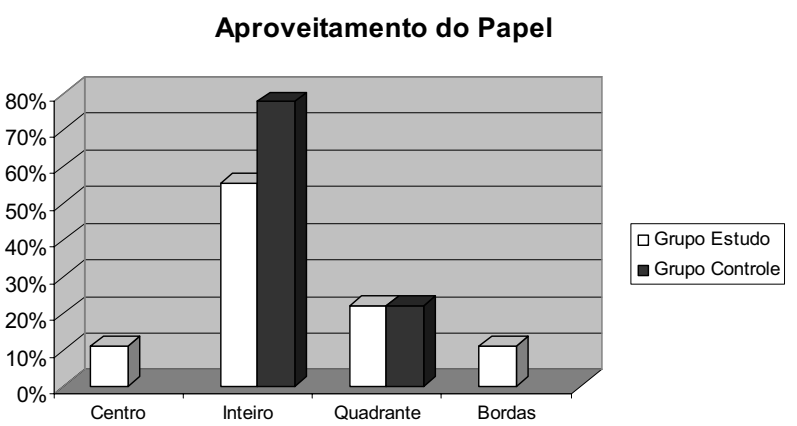

Figura 4 - Distribuição das crianças quanto ao aproveitamento do papel

Quanto à proporção dos objetos no desenho (Figura 5) observou-se que no grupo estudo, três crianças $(48,86 \%)$ realizaram seus desenhos utilizando proporção adequada entre os objetos e qua-

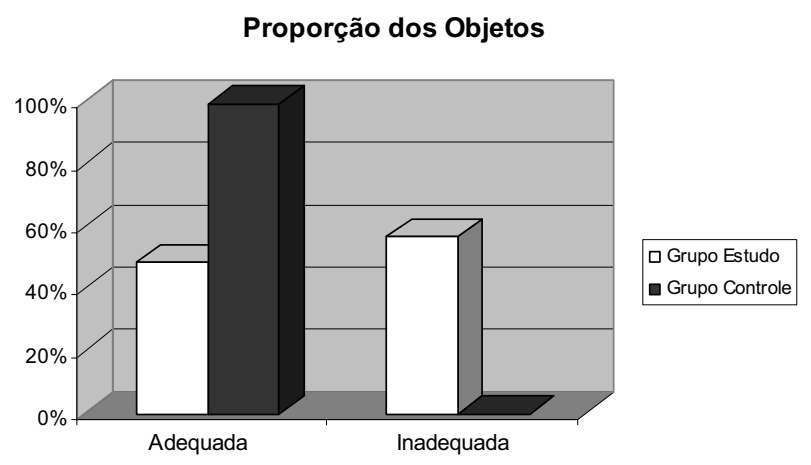

Figura 5 - Distribuição das crianças quanto à proporção dos objetos no desenho

tro crianças $(57,14 \%)$ realizaram seus desenhos com proporção inadequada entre os objetos; já no grupo controle, observou-se que nove crianças $(100 \%)$ realizaram o desenho utilizando proporção adequada entre os objetos.

Conforme mostra a Figura 6, constata-se que, quanto ao aspecto de tamanho, na primeira aplicação do teste o grupo estudo obteve média de acerto de $98,14 \%$ e o grupo controle $92,44 \%$. Na repetição do teste, o grupo estudo obteve $50 \%$ de média de acerto e o grupo controle $83 \%$.

Considerando o aspecto de espessura (Figura 7) verifica-se que, na primeira aplicação do teste, o grupo estudo obteve média de acerto de $81,28 \%$ e o grupo controle $85 \%$. Na repetição do teste, o grupo estudo obteve $87,32 \%$ de média de acerto e o grupo controle $83 \%$, fato que não evidencia diferenças significantes do ponto de vista clínico. 
Tamanho

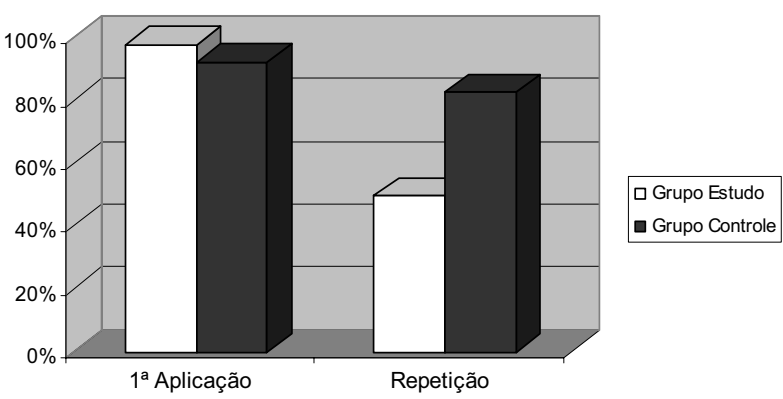

Figura 6 - Distribuição das crianças quanto à média de acerto nas tarefas que envolviam tamanho

Espessura

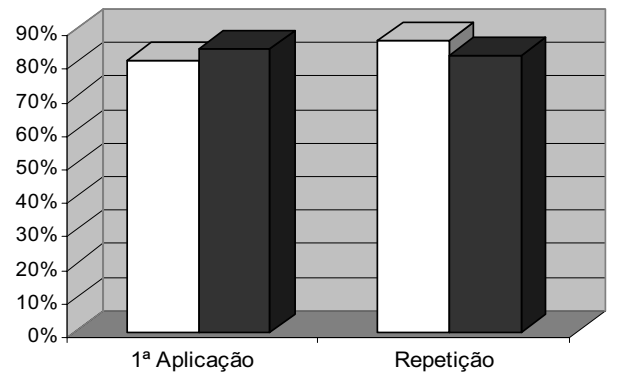

$\square$ Grupo Estudo

- Grupo Controle

Figura 7 - Distribuição das crianças quanto à média de acerto nas tarefas que envolviam espessura

Tamanho + Espessura

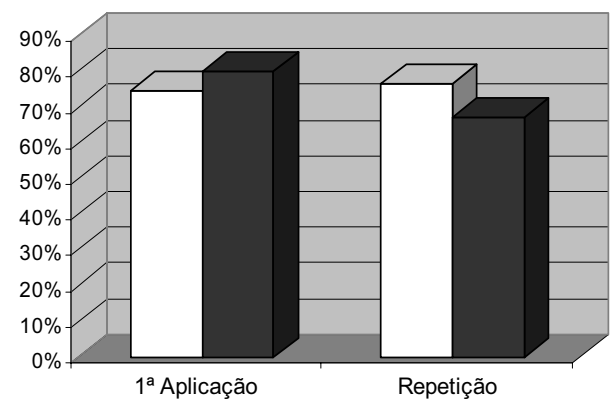

$\square$ Grupo Estudo

- Grupo Controle

Figura 8 - Distribuição das crianças quanto à média de acerto nas tarefas que envolviam espessura e tamanho

Observando a Figura 8 notou-se que, na primeira aplicação do teste de espessura e tamanho, o grupo estudo atingiu uma média de acerto de $75 \%$ e o grupo controle $80,55 \%$. Na repetição do teste, - grupo estudo atingiu uma média de acerto de $77,08 \%$ e o grupo controle $67,5 \%$.
Tamanho + Espessura + Posição Espacial

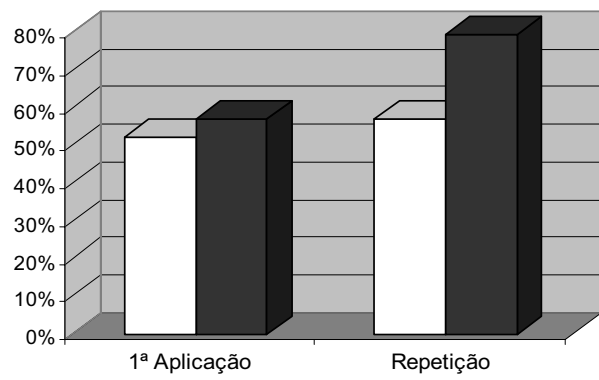

$\square$ Grupo Estudo Grupo Controle

Figura 9 - Distribuição das crianças quanto à média de acerto nas tarefas que envolviam espessura, tamanho e posição espacial

\section{Raw Score}

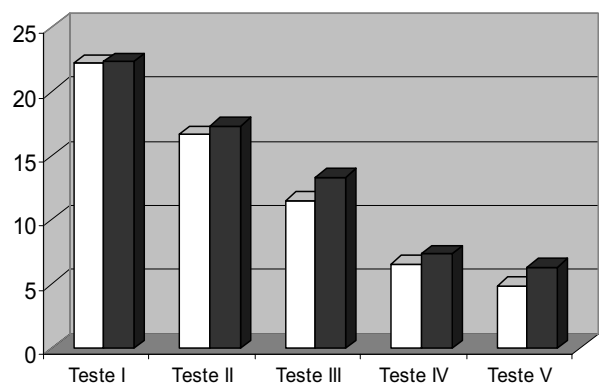

$\square$ Grupo Estudo - Grupo Controle

Figura 10 - Distribuição das crianças quanto à média do Raw Score obtido em cada teste

Em relação aos aspectos de espessura, tamanho e posição espacial (Figura 9) observou-se que, na primeira aplicação do teste, o grupo estudo atingiu uma média de acerto de $52,5 \%$ e o grupo controle $57,5 \%$. Já na repetição do teste, o grupo estudo atingiu uma média de $57.5 \%$ de acerto e o grupo controle $80 \%$.

Observando a Figura 10, verifica-se que praticamente não houve diferença quanto à média do Raw Score obtido em cada teste nos grupos avaliados, porém as crianças do grupo controle apresentaram melhor desempenho em todas as tarefas avaliadas.

Em relação ao Quociente Percentual (PQ) obtido pelo Teste Frostig (Figura 11) observa-se que o grupo estudo alcançou nota média de 96,25 e o grupo controle 101,75 , o que demonstra que houve apenas uma discreta diferença entre os dois grupos quanto aos aspectos avaliados neste teste (coordenação motora e visual, discriminação de figura-fundo, constância de forma, posição espacial e relações espaciais). 


\section{Quociente Perceptual}

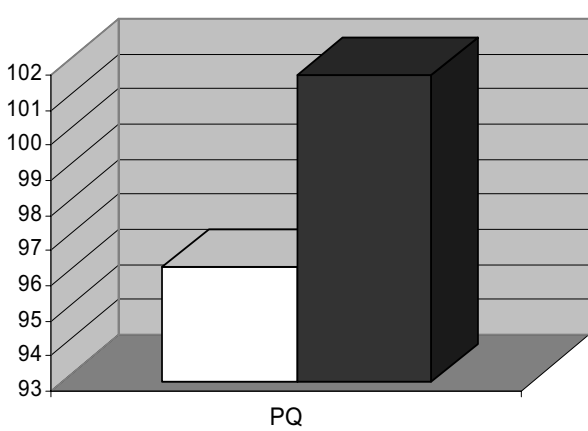

$\square$ Grupo Estudo - Grupo Controle

Figura 11 - Distribuição das crianças quanto à média do Quociente Perceptual (PQ) obtido no Teste Frostig

\section{DISCUSSÃO}

Pode-se verificar que não houve diferenças significativas do ponto de vista clínico, quanto ao desenvolvimento motor das crianças do grupo estudo com as do grupo controle (Tabela 3). Alguns estudos registraram em torno de $21 \%$ de crianças vestibulopatas com retardo no desenvolvimento motor, já que uma disfunção labiríntica, geralmente uma hipoatividade, pode contribuir para aumentar a média de idade para sentar e andar 5,10,11,20,32,33,40. Porém, a literatura também relata que, apesar da disfunção vestibular representar um risco para a aquisição de algumas habilidades motoras, tais como sentar, andar e na aquisição de respostas posturais adequadas, o fato da criança não apresentar atraso no desenvolvimento neuro-psicomotor, não descarta um distúrbio vestibular ${ }^{40}$.

Os sintomas e sinais mais comuns na vestibulopatia infantil podem ser cefaléia, tontura, alteração de comportamento, cinetose, distúrbio do sono, inquietude, choro sem etiologia, preferência por berço, otopatias, dores abdominais, desequilíbrio, anormalidades posturais, náusea, vômito, quedas, disacusia neurossensorial, nistagmo, ataxia, problemas escolares, atrasos motores, de desenvolvimento e distúrbios de linguagem (Tabelas 4 e 5) ${ }^{5,9-13,20-25,31-34,37,38,40,41}$.

A análise da repetição de ano letivo tornouse prejudicada devido à proposta de aprovação contínua adotado pelo atual sistema educacional (Tabela 5).

Apesar de acometimentos do sistema vestibular poderem influenciar algumas fases do comportamento infantil, pois este sistema juntamente com o SNC tem como função controlar a posição do corpo, os movimentos dos olhos e a percepção espacial que são importantes para a aquisição de muitas aprendizagens (como a linguagem falada e escrita) 5,10-13,20,23-36,40 não há diferença quanto ao número de fixações oculares entre crianças com distúrbio de leitura e escrita e crianças normais. O que pode justificar o interesse pela leitura em ambos os grupos estudados desta pesquisa (Tabela 5) ${ }^{32,42}$.

O sintoma de hipoacusia foi registrado em apenas 02 crianças, sendo uma pertencente ao grupo estudo e uma ao grupo controle, fato que concorda com a literatura estudada, já que o registro deste sintoma em crianças com vestibulopatia também não é constante, está em torno de 5,4\% ${ }^{40}$.

O sistema vestibular é sensível ao movimento no espaço e a mudanças posturais, contribuindo para a orientação espacial e para a coordenação motora. A utilização do hemisfério inferior da folha (borda inferior) parece se referir a uma necessidade concreta de apoio no mundo para as crianças se sentirem melhor equilibradas, já que a literatura relata que existe uma marca de "fragilidade" evidenciada nos desenhos infantis de crianças com vestibulopatia que podem ser representadas nas figuras humanas e na apresentação do desenho na folha que sugerem forte relação com a vivência do desequilíbrio (Figuras 3 e 4) 26,34,37,43.

$\mathrm{Na}$ Figura 5 pode-se verificar que crianças com distúrbios vestibulares podem ter, além de sensações distorcidas de seu próprio tamanho, sensações distorcidas do tamanho de objetos à distância e das relações espaciais. Já que, é o sistema vestibular que controla a posição do corpo, os movimentos dos olhos e a percepção espacial visando à manutenção do equilíbrio corporal ${ }^{24,44}$.

Segundo a literatura, noções referentes ao tamanho se referem ao espaço e estas noções de espaço são adquiridas pela organização proprioceptiva, vestibular, visual e cerebelar (Figura 6) ${ }^{25}$.

Também é descrito que, crianças com comprometimento vestibular têm padrões anormais de desenvolvimento e necessitam de atividades especiais para favorecer ou melhorar seus controles posturais, de equilíbrio, oculomotores e visuais, assim como para melhorar sua coordenação viso-manual. Além disso, pacientes com vertigem visual têm maiores respostas posturais para desorientação visual no meio ambiente, discordando dos achados que podem ser observados nas Figuras 7 e $8^{28,45}$.

O sistema vestibular controla a posição do corpo, os movimentos dos olhos e a percepção espacial com a finalidade de manter o equilíbrio corporal. O sistema postural atua sobre os mecanismos de aprendizagem por meio de integrações sensoriais provenientes fundamentalmente de aferências proprioceptivas, vestibulares e visuais. Ele permite, direta ou indiretamente, a percepção da noção espa- 
cial e temporal nas sucessivas etapas do desenvolvimento (Figura 9) 4,5,10-13,22-25,27,28,31-34,36,38,40,41.

Os resultados deste item podem ter sido influenciados pela extensão do teste que tornavam as crianças pequenas cansadas e desatentas rapidamente, o que dificultou à interpretação das atividades realizadas.

É importante destacar que cinco crianças, de ambos os grupos, acima de dez anos de idade, apresentaram desempenho insatisfatório em algum item das cinco tarefas exigidas no Teste Frostig, portanto, segundo a proposta do teste, pode-se considerar que essas crianças possuem dificuldades em relação ao conteúdo abordado, por este motivo, não foram enquadradas na contagem do Quociente Perceptual. Sendo assim o Quociente Perceptual não foi calculado para estas crianças, visto que, elas já foram consideradas alteradas, pois o score mínimo nas tarefas não foi alcançado.

\section{CONCLUSÃO}

Os resultados demonstraram que crianças com afecções vestibulares periféricas, ao desenhar, aproveitam o papel de forma menos homogênea do que crianças sem evidências de afecções vestibulares. Além disso, crianças com vestibulopatia apresentam maior dificuldade para desenhar figuras humanas e proporção adequada entre os objetos presentes no desenho, quando comparadas às crianças sem evidências de vestibulopatia.

Portanto, é de extrema importância que seja realizado o diagnóstico das afecções vestibulares, da forma mais precoce possível, para que o tratamento / reabilitação seja iniciado rapidamente e sintomas como a desorientação espacial, não influenciem futuras aprendizagens da criança.

\begin{abstract}
Purpose: to check how is the child's spatial perception in vestibular disorder. Methods: prospective study of 18 children, between 3 and 15-year old, under treatment in Ambulatório de Otorrinolaringologia/ Hospital das Clínicas da Faculdade de Medicina da Universidade de São Paulo. Space perception was assessed through a free activity (free drawing) and two directed activities: the Frostig test and an adaptation of the Token Test. Of the 18 evaluated children, 09 composed the study group (diagnosed as having a vestibular disorder) and 09 composed the control group (with no history of vestibular disorder or dizziness). Children who showed central nervous system impairments were excluded. Results: in relation to the free drawing, $77.78 \%$ of the control group and $55.55 \%$ in the study group used the whole sheet of paper provided. Regarding the proportion of the objects, $100 \%$ of the control group showed an adequate proportion in their drawings and it was found in $48.86 \%$ of the study group. In the adapted Token Test, the study group showed a major difficulty. In the Frostig test, a small difference between the groups in the Perceptual quotient was observed. Conclusion: the children with vestibular disorders, while drawing, used the sheet of paper in a less homogeneous way, showing more difficulty to draw human pictures and an inadequate proportion among the drawn objects. In this way, it is important that the vestibular diagnosis should be done the earliest possible in order to anticipate the treatment/rehabilitation. This may prevent the impairment of spatial perception from negatively influencing the learning process.
\end{abstract}

KEYWORDS: Dizziness; Vertigo; Child; Space Perception

\section{REFERÊNCIAS}

1. Luxon LM. The medical management of vertigo. J Laryngol Otol. 1997; 111(12):1114-21.

2. Pedalini MEB, Bittar RSM. Reabilitação vestibular: uma proposta de trabalho. Pró-Fono. 1999; 11(1):140-4.

3. Oliveira JAA. Fisiologia clínica do equilíbrio. In: Costa SS, Cruz OLM, Oliveira JAA. Otorrinolaringologia: princípios e prática. Porto Alegre: Artes Médicas; 1994. p. 66-78.
4. Ganança MM, Caovilla HH. A vertigem e sintomas associados. In: Ganança MM, Vieira RM, Caovilla $\mathrm{HH}$. Princípios de otoneurologia. São Paulo: Atheneu; 1998. p. 3-5.

5. Ganança MM. Da vestibulometria em crianças com distúrbios de linguagem [doutorado]. São Paulo (SP): Escola Paulista de Medicina; 1989.

6. Northern JL, Downs MP. Desenvolvimento do comportamento auditivo. In: Audição em crianças. 3. ed. São Paulo: Manole; 1989. p. 101-41. 
7. Barbosa MSM, Ganança FF, Caovilla HH, Ganança MM. Reabilitação labiríntica: o que é e como se faz. RBM-ORL. 1995; 2(1):24-34.

8. Douglas CR. Fisiologia do equilíbrio. In: Douglas CR. Tratado de fisiologia aplicada à fonoaudiologia. São Paulo: Robe; 2002. p. 193-208.

9. Ramos MEVC, Azevedo APM, Lengruber A, Campos A, Chiari EP. Roteiro de avaliação otoneurológica infantil do recém-nato até a criança de 12 anos. RBM-ORL. 1997; 4(5):164-8.

10. Medeiros IRT. Vestibulopatias na infância. In: Bittar RSM, Sanches TG, Formigoni LG. Temas de otoneurologia. v. 3. Apostila da Clínica de Otorrinolaringologia do Hospital das Clínicas da Faculdade de Medicina da Universidade de São Paulo. 1998 [apostila]. p. 29-35.

11. Medeiros IRT, Bittar RSM, Pedalini MEB, Lorenzi MC, Kii MA, Formigoni LG. Avaliação do tratamento dos distúrbios vestibulares na criança através da posturografia dinâmica computadorizada: resultados preliminares. J Pediatr. 2003; 79(4):337-42.

12. Ganança MM, Caovilla HH, Munhoz MSL, Munhoz ML, Ganança FF. Tratamento da vertigem na criança. Pediatr Modern. 1997; 33(1/2):7-22.

13. Riina N, Ilmari $P$, Kentala E. Vertigo and imbalance in children: a retrospective study in Helsinki University otorhinolaryngology clinic. Arch Otolaryngol Head Neck Surg. 2005; 131(11):996-1000.

14. Rodgers GK, Telischi FF. Meniere's disease in children. Otolaryngol Clin North Am. 1997; 30(6):1101-4.

15. Golz A, Netzer A, Angel-Yeger B, Westerman ST, Gilbert LM, Joachims HZ. Effects of middle ear effusion on the vestibular system in children. Otolaryngol Head Neck Surg. 1998; 119(6):695-9.

16. Sadé J, Yaniv E. Meniere's disease in infants. Acta Otolaryngol. 1984; 97(1-2):33-7.

17. Choung YH, Park K, Kim CH, Kim HJ, Kim K. Rare cases of Ménière's disease in children. J Laryngol Otol. 2006; 120(4):343-52.

18. Balatsouras DG, Kaberos A, Assimakopoulos D, Katotomichelakis M, Economou NC, Korres SG. Etiology of vertigo in children. Int $\mathrm{J}$ Pediatr Otorhinolaryngol. 2007; 71(3):487-94.

19. Choung YH, Park K, Moon SK, Kim CH, Ryu SJ. Various causes and clinical characteristics in vertigo in children with normal eardrums. Int J Pediatr Otorhinolaryngol. 2003; 67(8):889-94.

20. Bower CM, Cotton RT. The spectrum of vertigo in children. Arch Otolaryngol Head Neck Surg. 1995; 121(8):911-5.

21. Erbek SH, Erbek SS, Yilmaz I, Topal O, Ozgirgin N, Ozluoglu LN, Alehan F. Vertigo in childhood: a clinical experience. Int J Pediatr Otorhinolaryngol. 2006; 70(9):1547-54.
22. Formigoni LG. A avaliação vestibular na criança. In: Ganança MM. Vertigem tem cura? O que aprendemos nestes últimos 30 anos. São Paulo: Lemos; 1998. p. 117-26.

23. Ganança FF, Ganança CF. Vertigem na infância e na adolescência. In: Ganança MM. Vertigem tem cura? O que aprendemos nestes últimos 30 anos. São Paulo: Lemos; 1998. p. 37-47.

24. Formigoni LG, Medeiros IRT, Santoro PP, Bittar RSM, Bottino MA. Avaliação clínica das vestibulopatias na infância. Rev Bras Otorrinolaringol. 1999; 65(1):78-82.

25. Quirós JB, Cella MD. Exploración de la noción espacial. In: Quirós JB, Cella MD. La dislexia em la niñez. Buenos Aires: Paidos; 1965. p. 153-8.

26. Quirós JB, Götter R. Importância de la imagen corporal en el estudio del lenguaje. In: Quirós JB, Götter R. El lenguaje en el niño. Buenos Aires: Casa Ares; 1973. p. 137-72.

27. Quirós JB, Cella MD. Diagnostico de sordera infantil y sindromes vestibulares o afasoideos que possibilitan su confusion. In: Quirós JB, Cella MD. La audiometria del adulto y del niño. Buenos Aires: Paidos; 1974. p. 388-402.

28. Quirós JB, Schrager OL. Sistema postural, potencialidad corporal y lenguaje. In: Quirós JB. El Lenguaje lectoescrito y sus problemas. Buenos Aires: Medica Panamericana; 1977. p. 84-96.

29. Quirós JB, Schrager OL. Entrenamiento postural y motor em lãs perturbaciones de aprendizaje de origen propioceptivovestibular. In: Quirós JB, Schrager OL. Lenguaje, aprendizaje y psicomotricidad. Buenos Aires: Medica Panamericana; 1979. p. 203-31.

30. Quirós JB, Schrager OL. Experiencias sobre postura y aprendizaje: determinación de los síndromes propioceptivovestibulares. In: Quirós JB, Schrager OL. Lenguaje, aprendizaje y psicomotricidad. Buenos Aires: Medica Panamericana; 1979. p. 171-89.

31. Ganança MM, Caovilla HH. Labirintopatia na infância. In: Caldas N, Sih T. Otologia e audiologia em pediatria. Rio de Janeiro: Revinter; 1999. p. 277-86.

32. Ganança CF, Pupo AC, Caovilla HH, Ganança MM. Disfunção vestibular em crianças e adolescentes com mau rendimento escolar. Fono Atual. 2000; 11:21-7.

33. Campos MI, Ganança FF, Caovilla HH, Ganança MM. Prevalência de sinais de disfunção vestibular em crianças com vertigem e/ou outros tipos de tontura. RBM-ORL. 1996; 3(3):165-71.

34. McHugh HE. Auditory and vestibular disorders um children. Laryngoscope. 1962; 72:555-65.

35. Schrager OL. Visión, sistema postural y aprendizaje. In: Quirós JB. El lenguaje lectoescrito y sus 
problemas. Buenos Aires: Medica Panamericana; 1977. p. 97-114.

36. Caovilla HH. O equilíbrio corporal e os distúrbios da comunicação humana. In: Ganança MM, Vieira RM, Caovilla HH. Princípios de otoneurologia. São Paulo: Atheneu; 1998. p. 1-2.

37. Santos RMR, Medeiros IRT, Bittar RSM. Aspectos emocionais da criança portadora de vestibulopatias: achados preliminares baseados em desenhos e histórias. Arq Otorrinolaringol. 2003; 7(4):278-82.

38. Ganança MM, Caovilla HH. Correlação entre distúrbio de linguagem e disfunção labiríntica. Pediatr Modern. 1990; 25(4):128-45.

39. De Renzi E, Vignolo LA. The token test: a sensitive test to detect receptive disturbances in aphasics. Brain. 1962; 85:665-78.

40. Soares IP, Aguirre BR, Formigoni LG. Vertigem na infância. Rev Bras Otorrinolaringol. 1994; 60(2):92-7.
41. Ganança MM, Caovilla HH, Munhoz MSL, Ganança FF. Tonturas na criança e no adolescente. RBM-ORL. 1995; 2(4):217-42.

42. Santos MTM, Behlau MS, Caovilla HH. Crianças com distúrbio de leitura e escrita: movimentos oculares na leitura à nistagmografia computadorizada. RBM-ORL. 1995; 2(2):100-7.

43. Kileny P. Avaliação da função vestibular. In: Katz J. Tratado de audiologia clínica. São Paulo: Manole; 1989. p. 592-612.

44. Zeigelboim BS, Pereira LD, Barbosa MSM, Ito YI, Tavares AR, Caovilla HH. Alterações centrais de vias auditivas e vestibulares em um caso de distúrbio de linguagem. RBM-ORL. 1995; 2(6):454-9.

45. Guerraz M, Yardley P, Bertholon P, Pollak L, Rudge P, Gresty MA, Bronstein AM. Visual vertigo: symptom assessment, spatial orientation and postural control. Brain. 2001; 124(8):1646-56. 